\title{
Data Universal Numbering System
}

National Cancer Institute

\section{Source}

National Cancer Institute. Data Universal Numbering System. NCI Thesaurus. Code C134003.

A proprietary system developed and regulated by Dun \& Bradstreet that assigns a unique nine digit numeric identifier to a single business entity location. 